\title{
Bridged Refinement for Transfer Learning
}

\author{
Dikan Xing, Wenyuan Dai, Gui-Rong Xue, and Yong Yu \\ Department of Computer Science and Engineering \\ Shanghai Jiao Tong University, Shanghai, China \\ \{xiaobao, dwyak, grxue, yyu\}@apex.sjtu.edu.cn
}

\begin{abstract}
There is usually an assumption in traditional machine learning that the training and test data are governed by the same distribution. This assumption might be violated when the training and test data come from different time periods or domains. In such situations, traditional machine learning methods not aware of the shift of distribution may fail. This paper proposes a novel algorithm, namely bridged refinement, to take the shift into consideration. The algorithm corrects the labels predicted by a shift-unaware classifier towards a target distribution and takes the mixture distribution of the training and test data as a bridge to better transfer from the training data to the test data. In the experiments, our algorithm successfully refines the classification labels predicted by three state-of-the-art algorithms: the Support Vector Machine, the naïve Bayes classifier and the Transductive Support Vector Machine on eleven data sets. The relative reduction of error rates is about $50 \%$ in average.
\end{abstract}

\section{Introduction}

Supervised learning requires enough, if not many, high-quality labeled examples to guide the learning progress for a model, by which we predict the labels of newly coming test data. Labeling examples are labor-intensive, and what makes things worse is that more and more labeled data become out of date as time goes by. For example, in the past years, there are a large number of textual data on the Web such as news reports that were written in formal style. But recently, blogs have been emerging, and their owners begin to write their posts in a style increasingly different from what they read in news reports. Past labeled news data thus cannot be used to reliably classify blog articles, since the usage of vocabulary becomes different in blog articles from news articles.

Transfer learning focuses on how to utilize those data from different time periods or domains to help the current learning task. Many previous researches on transfer learning are for so-called "multi-task" learning, where there are $K$ tasks at hand and one wants to complete the $K$-th task, the mainly-focused task, with the help of the previous $K-1$ tasks.

In this paper, we focus on such a situation that we only have one task, by which we mean that the set of target categories are fixed, but the document-marginal distribution, $P(d)$, of the training data $\mathcal{L}$ and the test data $\mathcal{U}$ are different, for 


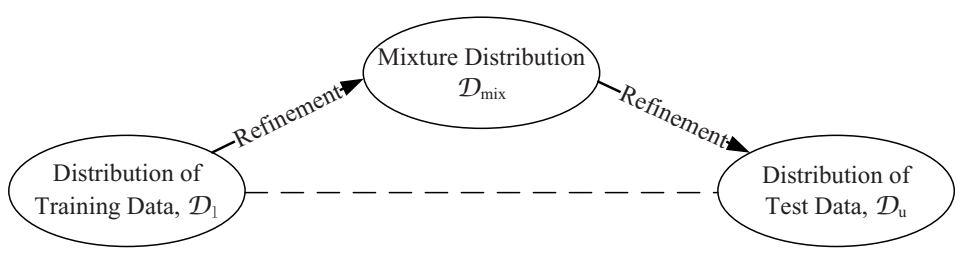

Fig. 1. Bridged Refinement

example being $\mathcal{D}_{l}$ and $\mathcal{D}_{u}$ respectively. We only have labeled data governed by $\mathcal{D}_{l}$, i.e. the training data $\mathcal{L}$, and have no labeled data governed by $\mathcal{D}_{u}$. All test data, $\mathcal{U}$, which are governed by $\mathcal{D}_{u}$ are to be classified. We want to transfer the knowledge in the training data $\mathcal{L}$ to well classify the test data $\mathcal{U}$.

To achieve this goal, in this work, we propose a bridged refinement algorithm. The algorithm receives the predictions from a shift-unaware classifier, which is trained from data governed by $\mathcal{D}_{l}$ and is expected to work well on data governed by $\mathcal{D}_{l}$. But now the predictions produced by it may be far from satisfactory since the distribution of the test data changes from $\mathcal{D}_{l}$ to $\mathcal{D}_{u}$. Our algorithm refines the classification labels in a two-step way, firstly towards the mixture distribution $\mathcal{D}_{\text {mix }}$ and secondly towards the test distribution $\mathcal{D}_{u}$. The mixture distribution $\mathcal{D}_{\text {mix }}$ governs both the training and test data as a whole. Hence $\mathcal{D}_{\text {mix }}$ is more similar both to $\mathcal{D}_{l}$ and to $\mathcal{D}_{u}$ than $\mathcal{D}_{l}$ to $\mathcal{D}_{u}$, so regarding it as an intermediate step, or a bridge, makes the two steps of refinement relatively easy. The bridged transfer process is intuitively depicted in Figure 1.

In each step of refinement, we want to refine the classification labels to make them more consistent under the target distribution (i.e. $\mathcal{D}_{\text {mix }}$ in the first step and $\mathcal{D}_{u}$ in the second). Considering the observation that two identical documents even under two different distributions, e.g. news vs. blogs, are supposed to be in the same category, the conditional distribution of the class label $c$ on the input $d, P(c \mid d)$, does not vary. Based on this assumption 1 , our refinement operation tries to make the labels more consistent under the target distribution.

The algorithm aims at refining classification labels instead of the decision boundary of a model. Refining a model seems more useful since it can be later used for newly coming test data. But it may be difficult in our problem, because what can be transferred from the labeled training data $\mathcal{L}$ totally depends on the test data $\mathcal{U}$. Different test data may require different knowledge to be transferred. Hence it is more realistic to set our goal to better classifying only the currentlygiven test data rather than learning or refining a universal classifier model, so what we are to solve in this paper is to refine the classification labels instead of the classifier itself.

The experimental results show that our bridged refinement algorithm can successfully refine results predicted from different kinds of shift-unaware classifiers, reducing the error rates by $50 \%$ in average compared with those classifiers. We attribute the success of our algorithm to the awareness of distribution

$\overline{{ }^{1} \text { More details }}$ are available at http://ida.first.fraunhofer.de/projects/different06/. 
shift in each refinement step and the utilization of the mixture distribution as a bridge.

The rest of this paper is organized as follows. Some related work is discussed in Section 2. Our bridged refinement algorithm is described in Section 3 and the experiments are reported in Section 4. In Section 5, we conclude our work and mention some future work, as well as several interesting thoughts for extension.

\section{Related Work}

Utilizing labeled data from different domains, tasks or distributions as auxiliary data for a primary learning task is discussed in the transfer learning context 1211310. 1] provided a theoretical justification of transfer learning through multi-task learning.

However, there are two slight differences between our work and many previous researches in the transfer learning literature. Firstly they usually utilize the auxiliary data to bias the classifier while our work focuses on refinement of classification results of the test data instead of the classifier itself, which is like what transductive learning does, as Vapnik [15] said,

When solving a problem of interest, do not solve a more general problem as an intermediate step. Try to get the answer that you really need but not a more general one.

What we really need here is the correct labels for those test data, so we do not refine the model as an intermediate step, which is more general and more difficult, but directly refine the classification labels. Secondly, many researches require confidently labeled data under the new distribution, albeit little in amount, while ours do not need any.

The sample selection bias problem was mentioned in e.g. 16. In that paper, the author concluded four kinds of biases, based on the dependency and independency between whether one example is selected and its feature-label pair $(x, y)$. The problem we solve in this paper is similar to the second kind, which is, whether a training example is picked up (e.g. out-of-date labeled data vs. new data) is independent of the response $y$ (positive or negative) given the input $x$. The author also suggested a solution to this kind of bias provided that given the input $x$, the selection criterion can be modeled properly, which is not needed in our work.

The refinement step in our proposed algorithm is suggested by the PageRank algorithm 9. The PageRank algorithm conveys a mutual reinforcement principle that good pages may also link to some other good pages, thus it yields

$$
P R=M^{T} \times P R
$$

where $P R$ is a vector, each element of which is the score of each page, and $M$ is the adjacent matrix of pages with each row $L_{1}$-normalized. Under a random surfer model, the formula is appended with another vector, $E$, to reflect the fact 
that the surfer may be bored with clicking through hyper-links in the Web pages and input a completely new URL in the browser:

$$
P R=\alpha M^{T} \times P R+(1-\alpha) E
$$

Such modification also involves some computational consideration such as rank sinks [2] and convergence to a unique point. Meanwhile a lot of researches regard the extra item $E$ as a complement to the mutual reinforcement principle. The tradeoff between the two factors are controlled by the teleportation coefficient $\alpha$. This kind of work includes: Topic-sensitive PageRank [5], TrustRank [4], etc.

\section{Bridged Refinement Algorithm}

Our algorithm receives the predictions from a shift-unaware classifier and then makes two-step refinements on these predictions, taking the mixture distribution $\mathcal{D}_{\text {mix }}$ as a bridge.

\subsection{Refinement}

One run of refinement is to correct the labels of the documents to make them more consistent with each other under the distribution observed in the documents themselves, which we name as the target distribution. In the first (left) refinement in Figure 1 the target distribution is $\mathcal{D}_{\text {mix }}$, observed in $\mathcal{L} \cup \mathcal{D}$, and in the second one, it is $\mathcal{D}_{u}$, observed in $\mathcal{U}$.

In order to clarify what we mean by "consistent", we introduce our assumption first. Formally, we assume that the conditional probability of a specified class given a document $d, P(c \mid d)$, does not vary among different distributions: $P_{\mathcal{D}_{u}}(c \mid d)=P_{\mathcal{D}_{m i x}}(c \mid d)=P_{\mathcal{D}_{l}}(c \mid d)$, although the probability of a document $P(d)$ varies. This is based on such an observation that if identical documents appear both in the training data $\mathcal{L}$ and the test data $\mathcal{U}$, the labels should be the same. Taking it a step further, if one's neighbors have high confidence scores to belong to a specified category, the document itself may also receive a high confidence score to that category. This situation constitutes a mutual reinforcement relationship between documents. This kind of influence may occur across the decision boundary found by a shift-unaware classifier and thus can be used to correct the labels.

In a word, by the term "consistent", we mean similar documents should have close confidence values to the same category, where the similarity is measured in some way under the target distribution.

We now introduce the algorithm for refining the confidence score of each document to belong to a specified category under the target distribution. Mathematically, let $M$ denote the adjacent matrix of documents, where $M_{i j}$ is set to 0 if $d_{j}$ is not a neighbor of $d_{i}$ and $1 / K$ if $d_{j}$ is a neighbor of $d_{i}$, where $K$ is the number of neighbors. The information of distribution governing the documents is, in some way, captured by the matrix $M$. 
Input: The document collection $D$ in bag-of-words representation, the unrefined confidence score $U C o n f_{i, j}$ of each document $d_{i}$ and each category $c_{j}$, and $\alpha, K$

Output: The refined confidence scores $R$ Conf

1 foreach Pair of documents $d_{i}, d_{j}$ do

$2 \mid \operatorname{dist}(i, j)=1-\cos \left(d_{i}, d_{j}\right)$

3 end

4 foreach Document $d_{i}$ do

$5 \quad$ Heap sort $\{\langle j, \operatorname{dist}(i, j)\rangle\}$ on $\operatorname{dist}(i, j)$ until top $K+1$ are sorted

$6 \quad N_{i} \leftarrow K$ nearest neighbors of document $d_{i}$ (excluding $d_{i}$ itself)

7 end

8 foreach Category $c_{j}$ do

$9 \quad U \operatorname{Conf} \cdot, j \leftarrow U \operatorname{Conf} \cdot, j /\left\|U \operatorname{Conf} f_{\cdot, j}\right\|_{1}$

10 end

11 repeat

12 foreach Document $d_{i}$ do

13 foreach Category $c_{j}$ do

14||$_{\text {end }} \operatorname{RConf}_{i, j}^{(t+1)} \leftarrow \alpha \sum_{s \in N_{i}} \operatorname{RConf}_{s, j}^{(t)} /\left|N_{i}\right|+(1-\alpha) U \operatorname{Conf} f_{i, j}$

16 end

17 until RConf converges ;

18 return $R$ Conf

Algorithm 1. Refinement Algorithm

$U C o n f_{\cdot, j}$ is a vector, each element of which denotes the confidence score of a document to belong to the category $c_{j}$ reported by those shift-unaware classifiers. Let $R C$ on $f_{\cdot, j}$ denote the refined confidence scores of each document to belong to the category $c_{j}$. The mutual reinforcement principle yields the following equation for solving $R$ Conf.,j:

$$
\operatorname{RConf}_{\cdot, j}=\alpha M^{T} \operatorname{RConf}_{\cdot, j}+(1-\alpha) U \operatorname{Conf} \cdot, j
$$

where $\alpha$ is the trade-off factor between the refinement process and the original (unrefined) confidence scores.

The equation can be solved in a closed form

$$
R_{\text {Conf }}, j=(1-\alpha) \times\left(I-\alpha M^{T}\right)^{-1} U \operatorname{Conf} \cdot, j
$$

or in an iterative manner as what is done in Algorithm 1 if $M$ is too large to be inversed efficiently.

$R C o n f_{\cdot, j}$ can also be explained in a random surfer model. Suppose the surfer wants to read documents of the category $c_{j}$, he/she starts at a random place and then reads similar documents until he/she decides to switch to a very different document from the one being read, but of the same category. The $i$-th element in $R$ Con $f_{\cdot, j}, R C o n f_{i, j}$, is the probability that the surfer may read $d_{i}$. RCon $f_{\cdot, j}$ tells the relatedness of each document to the category $c_{j}$. In other words, it can be regarded as an estimate to $P\left(d_{i} \mid c_{j}\right)$. 
Input: The document collection $\mathcal{L}$ and $\mathcal{U}$ in bag-of-words representation, the unrefined confidence score $U C \operatorname{Con} f_{i, j}$ of each document $d_{i}$ and each category $c_{j}$, and $\alpha, K$

Output: The refined labels of $\mathcal{U}$

1 Call Algorithm 1 with $D=\mathcal{L} \cup \mathcal{U}, U \operatorname{Conf}, \alpha$, and $K$. RConf $f^{1}$ is returned.

2 Call Algorithm 1 with $D=\mathcal{U}, R C o n f^{1}$ projected on $\mathcal{U}, \alpha$, and $K$. RConf $f^{2}$ is returned.

3 foreach Document $d_{i}$ in $\mathcal{U}$ do

$4 \quad$ cat $_{i}=\arg \max _{j} R \operatorname{Ron} f_{i, j}^{2}$

5 end

6 return cat $_{i}$ for all documents in $\mathcal{U}$.

Algorithm 2. Bridged Refinement Algorithm

The refinement step can be also regarded as a thinker. Given a document, he/she seeks from his/her memory for similar documents whatever they are new or old, and transfer the confidence of those similar articles to give a refined confidence score.

The refinement algorithm is shown in Algorithm 1. Line 1 to 3 compute the pairwise distances and in Line 4 to 7 neighbor sets are calculated. Line 8 to 10 normalize each $U$ Con $f_{\cdot, j}$ to unit length. The iterative manner for solving Equation [3 is implemented in Line 11 to 17. The refined results are returned in the last line. It should be noticed that the operations in Line 8 to 17 can be accomplished in very neat codes of a matrix programming language such as Matlab and that most part of the refinement algorithm can be easily adapted to be performed in a parallel way.

\subsection{Bridged Refinements}

The refinement step is mainly controlled by the examples governed by the target distribution, reflected in the adjacent matrix $M$ in Algorithm 11 Alternatives of refinements include refining under the mixture distribution $\mathcal{D}_{\text {mix }}$, or directly the target distribution $\mathcal{D}_{u}$. But in our algorithm, we work in a "bridged" way that we first refine under $\mathcal{D}_{\text {mix }}$ and then $\mathcal{D}_{l}$. Algorithm 2 gives the full description of our bridged refinement algorithm.

The mixture distribution $\mathcal{D}_{\text {mix }}$ observed by $\mathcal{L} \cup \mathcal{U}$ is similar both to $\mathcal{D}_{l}$ and to $\mathcal{D}_{u}$. Hence a relatively hard transfer from $\mathcal{D}_{l}$ to $\mathcal{D}_{u}$ is now bridged by $\mathcal{D}_{\text {mix }}$, by which we decompose a problem into two relatively easy ones. Algorithm 1 is performed twice. For the first time, we let $D$ be $\mathcal{L} \cup \mathcal{U}$, and for the second time, $D$ be $\mathcal{U}$. In this way, two different $M$ 's will be calculated in the two steps, reflecting the fact that each refinement is conducted towards a different distribution.

Finally we classify each document to one category that the final confidence in $R C o n f \cdot, j$ is the highest, which is done in Line 3 to 5 in Algorithm 2

$$
\text { cat }_{i}=\underset{c_{j}}{\arg \max } P\left(d_{i} \mid c_{j}\right)=\underset{j}{\arg \max } R \operatorname{Con} f_{i, j}
$$




\section{Experiment}

In this section, we want to verify the validity of the refinement step and the bridged way of refinements. We perform the experiments on two categories for the sake of simplicity, though it is straightforward to apply it for multi-categories.

\subsection{Data Preparation}

We prepare our data sets from three data collections: 20 Newsgroup 2 , SRAA 3 and Reuters-215784 for evaluating our bridged refinement algorithm.

These three data collections are not originally designed for transfer learning, so we need to make some modifications to make the distribution between the training data and the test data different. Each of these data sets has at least two level hierarchical structure. Suppose $A$ and $B$ are two root categories in one data collection, and $A_{1}, A_{2}$ and $B_{1}, B_{2}$ are sub-level categories of $A$ and $B$ respectively. Now we form the training and test data in this way. Let $A . A_{1}$, $B . B_{1}$ be the positive and negative examples in the training data respectively. Let $A . A_{2}, B . B_{2}$ be the positive and negative examples in the test data respectively.

Thus, the target categories are fixed, being $A$ and $B$, but the distributions of the training data and the test data are different but still similar enough for the evaluation of our refinement algorithm for transfer learning.

In SRAA, there are four discussion groups: simulated autos (simauto), simulated aviation (simaviation), real autos (realauto), real aviation (realaviation). We compose two data sets from SRAA. In 20 Newsgroups, there are seven top level categories, while three of them have no sub-categories. We compose six $\left(C_{4}^{2}\right)$ data sets from the remaining four categories. From Retures-21578, we compose three data sets. The detailed composition of these data sets are provided in the Appendix.

We make some preprocessing on the raw data, including turning all letters into lowercases, stemming words by the Porter stemmer [13, removing all stop words. According to 14, DF Thresholding can achieve comparable performance to Information Gain or CHI, but it is much easier to implement and less costly both in time and space complexity. Hence we use it to cut down the number of words/features and thus speed up the classification. The words that occur in less than three documents are removed. Then each document is converted into bagof-words presentation in the remaining feature space. Each feature value is the term frequency of that word in the document, weighted by its IDF $(\log N / D F) 5$

\subsection{Performance}

Working with Different Classifiers. To ensure that our bridged refinement algorithm for transfer learning is robust enough, we perform our algorithm on

\footnotetext{
$\overline{2}$ http://people.csail.mit.edu/jrennie/20Newsgroups/

${ }^{3}$ http://www.cs.umass.edu/ mccallum/data/sraa.tar.gz

${ }^{4}$ http://www.daviddlewis.com/resources/testcollections/reuters21578/

${ }^{5}$ We also conduct experiments on TF without IDF weighting. Both the unrefined and refined results are worse than that of the TFIDF representation.
} 


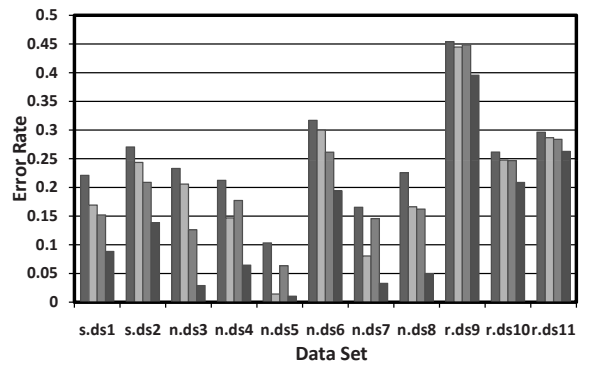

(a) Support Vector Machine

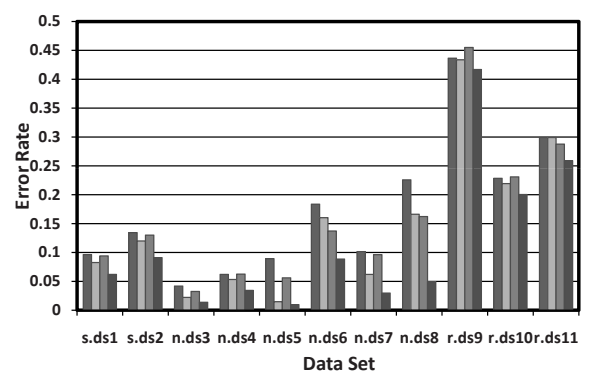

(c) Transductive Support Vector Machine

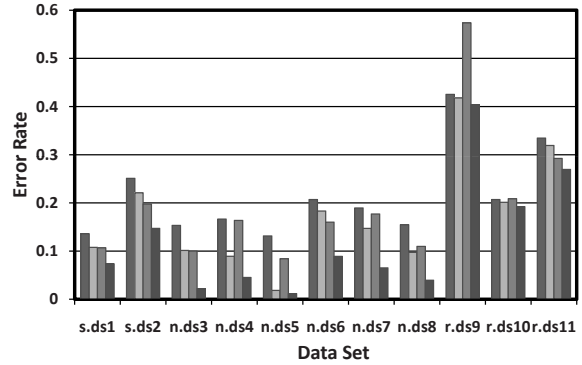

(b) Naïve Bayes Classifier

\begin{tabular}{|l}
\hline$\square$ Unrefined (leftmost) \\
$\square$ Refining directly on the \\
test distribution \\
$\square$ Refining on the mixture \\
distribution only \\
Bridged Refining \\
(rightmost)
\end{tabular}

Fig. 2. Error Rates on Eleven Data Sets

the labels predicted by three different shift-unaware classifiers, from which we receive the unrefined results: the Support Vector Machine 15, the naïve Bayes classifier [8] and the Transductive Support Vector Machine 7]. The Support Vector Machine is a state-of-the-art supervised learning algorithm. In our experiment, we use the implementation $\mathrm{SVM}^{\text {light }} 6$ with a linear kernel and all options set by default. The naïve Bayes classifier, despite its simplicity, performs surprisingly well in many tasks, so we also try to refine the classification results predicted by the naïve Bayes classifier. Last, we select a representative from semi-supervised learning algorithms, the Transductive Support Vector Machine, to see whether our refinement algorithm can reduce the error rate on that kind of learning algorithms. The experimental results show that, in all cases, our bridged refinement algorithm can reduce the error rates.

We first use each of the three models to learn from the training data $\mathcal{L}$ and classify the test data $\mathcal{U}$. Now we have labels for both the training data and the test data. For those positive examples, we set the corresponding element in $U C o n f .,+$ to 1 and for negative examples, we set to 0 . In binary classification, UConf.,- happens to be $\mathbf{1}-U C \operatorname{conf} f_{.,+}$. Then we pass these unrefined confidence scores to our algorithm and receive its outputs as the refined classification results.

Figure 2(a) shows the error rates of the Support Vector Machine on different data sets compared with the error rates of the refined results. In all data sets, our algorithm reduces the error rates. The greatest relative reductions of the error 
rates are achieved on $\mathrm{n} . \mathrm{ds} 5$ and $\mathrm{n} . \mathrm{ds} 3$, being $89.9 \%$ and $87.6 \%$ respectively. The average relative reduction of the error rates over all the eleven data sets is $54.3 \%$. Figure 2(b) shows the results about the naïve Bayes classifier. The greatest relative reductions of the error rates are achieved on $\mathrm{n} . \mathrm{ds} 5$ and $\mathrm{n} . \mathrm{ds} 3$, being $91.2 \%$ and $85.7 \%$. The average relative reduction is $51.5 \%$. Figure 2(c) shows the result about the Transductive Support Vector Machine. The greatest relative reductions of the error rates are achieved on $\mathrm{n} . \mathrm{ds} 5$ and $\mathrm{n} . \mathrm{ds} 8$, being $89.2 \%$ and $78.1 \%$ respectively. The average relative reduction is $45.4 \%$.

Does the Bridged Way Help? For each data set and classifier, we also experiment three different refinement strategies. They are (1) directly refining towards $\mathcal{D}_{u},(2)$ refining towards $\mathcal{D}_{\text {mix }}$ only, and (3) refining firstly towards $\mathcal{D}_{\text {mix }}$ and then towards $\mathcal{D}_{u}$.

Taking the Support Vector Machine as an example, each kind of refinement brings improvement in accuracy on all the eleven data sets. Moreover, still on all the eleven data sets, the bridged refinement outperforms another two one-step refinements consistently. Similar outperforming can be observed on another two classifiers. We attribute the success of the bridged refinement to the fact that the mixture distribution $\mathcal{D}_{\text {mix }}$ is more similar both to $\mathcal{D}_{l}$ and to $\mathcal{D}_{u}$ than $\mathcal{D}_{l}$ to $\mathcal{D}_{u}$ and thus the transfer is easy to perform.

\subsection{Parameter Sensitivity}

Our refinement algorithm includes two parameters, the number of neighbors $K$, and the teleportation coefficient $\alpha$. In this section we want to show that the algorithm is not that sensitive to the selection of these parameters.

We only report six series from all eleven data sets and three classifiers, that is in total 33 combinations, to make the figures clear and easy to read. The selected six series are composed of three groups, two in each working with a different classifier. The six series can also be decomposed into another three groups, two in each from a different collection. Such consideration makes the selected six data sets representative.

From Figure $3(\mathrm{a})$, we can find that the performance is not greatly sensitive to the selection of $K$ as long as $K$ is moderately large, so $K$ is empirically chosen as 70 in this paper. The teleportation coefficient $\alpha$ used in PageRank is reported to be 0.85 . From Figure $3(\mathrm{~b})$, we find 0.7 is a better choice. It is interesting to investigate the error rates when $\alpha$ is 0 or 1 . In the former case, the error rate is just that of the unrefined labels, while in the latter case, the labels predicted by those shift-unaware classifiers impose no impact on the final labels. In both cases, the performance is not that good.

Although the iterative manner for solving Equation 3 may require many iterations before convergence, in our experiments, we find that in our situation, convergence is reached within five iterations. Thus the iterative way is preferred to directly computing the closed form since inversing a large matrix requires much time. In Figure 3(c), Iteration 1 to 10 are performed for the first refinement towards $\mathcal{D}_{\text {mix }}$ and 11 to 20 are for the second refinement towards $\mathcal{D}_{u}$. 


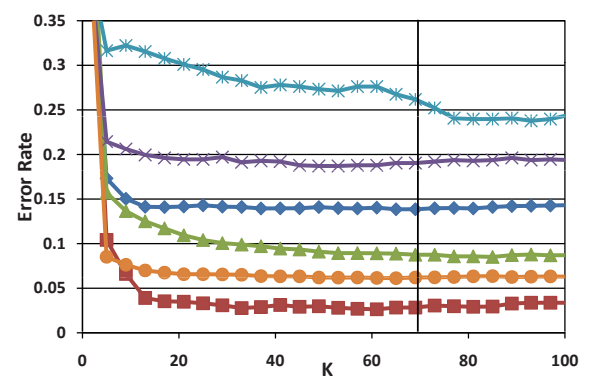

(a) Error Rate vs. $K$

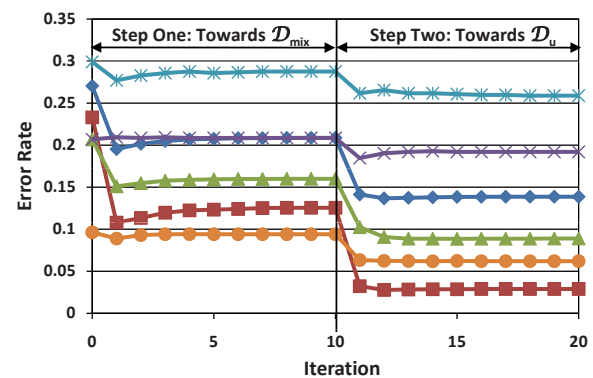

(c) Convergence

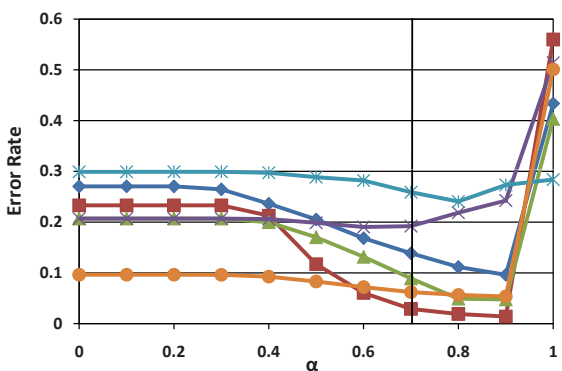

(b) Error Rate vs. $\alpha$

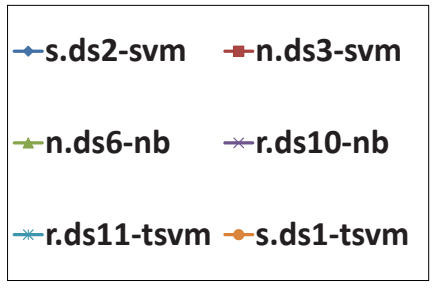

Fig. 3. Parameter and Iteration. (Points at $K=70$ in (a), at $\alpha=0.7$ in (b) and at Iteration $=20$ in (c) correspond to the same situations.)

\section{Conclusion and Future Work}

In this paper, we propose an algorithm to solve a problem in transfer learning, i.e. how to refine the classification labels predicted by a shift-unaware classifier when the distribution of inputs (documents), $P(d)$, varies from training data to test data, under the assumption that the conditional probability of the category given the document, $P(c \mid d)$, do not vary from training data to test data.

We concentrate on the refinement of the classification labels instead of the classification model, based on the consideration by Vapnik 15 that predicting test data when they are available at hand may be easier than first learning a model and then predicting.

To show the robustness of the algorithm, we refine the results produced by two supervised learning representatives, the Support Vector Machines and the naïve Bayes classifer, and one semi-supervised learning representative, the Transductive Support Vector Machine. In all cases, our refinement algorithm can reduce the error rate.

We also verify that taking the mixture distribution $\mathcal{D}_{\text {mix }}$ into account is successful since directly refining the results under the test distribution $\mathcal{D}_{u}$ is not as good as the two-step way. We attribute this to the fact that $\mathcal{D}_{\text {mix }}$ is more similar both to $\mathcal{D}_{l}$ and to $\mathcal{D}_{u}$ than $\mathcal{D}_{l}$ to $\mathcal{D}_{u}$ directly. 
There is a very interesting way to generalize our algorithm that can be tried. In our algorithm, only $(1-\lambda) \mathcal{D}_{l}+\lambda \mathcal{D}_{u}$, where $\lambda=0.5$ and 1 are considered, as $\mathcal{D}_{\text {mix }}$ and $\mathcal{D}_{u}$. Given a series: $\lambda_{0}=0, \lambda_{1}, \ldots, \lambda_{n-1}, \lambda_{n}=1$, where $\lambda_{i}<\lambda_{j}, i, j=$ $1, \cdots, n, i<j$, one can perform an $n$-step refinement instead of the two-step way in our algorithm, where $n=2$, and $\lambda_{1}=0.5, \lambda_{2}=1$. Thus the transfer process may be carried out in a more smooth way.

There are also some points in this work that can be handled more elegantly. For example, the unrefined confidence scores of both the training examples and the test examples are the same, but the labels of the test data are much less reliable than the training data. Therefore applying an discount to the confidence scores of the test data can be considered. Besides, given the refined confidence scores, the decision rule is based on the maximum likelihood principle. Other alternatives can be tried, including maximizing a posterior after modeling the category a prior probabilities, or directly assigning a document to a category where it ranks highest, etc. We will explore them further as our future work.

\section{Acknowledgements}

All authors are supported by a grant from National Natural Science Foundation of China (NO. 60473122). We thank the anonymous reviewers for their great helpful comments.

\section{References}

1. Ben-David, S., Schuller, R.: Exploiting task relatedness for multiple task learning. In: Proceedings of the Sixteenth Annual Conference on Learning Theory (2003)

2. Brin, S., Motwani, R., Page, L., Winograd, T.: What can you do with a web in your pocket. In: Bulletin of the IEEE Computer Society Technical Committee on Data Engineering, IEEE Computer Society Press, Los Alamitos (1998)

3. Caruana, R.: Multitask learning. Machine Learning 28(1), 41-75 (1997)

4. Gyongyi, Z., Garcia-Molina, H., Pedersen, J.: Combating web spam with trustrank. In: Proceedings of the 30th International Conference on Very Large Data Bases (2004)

5. Haveliwala, T.: Topic-sensitive PageRank. In: Proceedings of the Eleventh International World Wide Web Conference (2002)

6. Joachims, T.: Making large-scale SVM learning practical. In: Schölkopf, B., Burges, C., Smola, A. (eds.) Advances in Kernel Methods: Support Vector Learning, pp. 169-184. MIT Press, Cambridge (1999)

7. Joachims, T.: Transductive inference for text classification using Support Vector Machines. In: Bratko, I., Dzeroski, S. (eds.) Proceedings of the Sixteenthth Interantional Conference on Machine Learning, San Francisco, CA, USA, pp. 200-209 (1999)

8. Lewis, D.: Naive Bayes at forty: The independence assumption in information retrieval. In: Nédellec, C., Rouveirol, C. (eds.) Proceedings of European Conference on Machine Learning, Chemnitz, DE, pp. 4-15 (1998)

9. Page, L., Brin, S., Motwani, R., Winograd, T.: The PageRank citation ranking: bring order to the web, Stanford Digital Library Technologies Project (1998) 
10. Raina, R., Ng, A.Y., Koller, D.: Constructing informative priors using transfer learning. In: Proceedings of the Twentieth International Joint Conference on Artificial Intelligence (2006)

11. Schmidhuber, J.: On learning how to learn learning strategies. Technical Report FKI-198-94, Technische Universität München (1995)

12. Thrun, S., Mitchell, T.M.: Learning one more thing. In: Proceedings of the Fourteenth International Joint Conference on Artificial Intelligence (1995)

13. Porter, M.F.: An algorithm for suffix stripping. Program 14(3), 130-137 (1980)

14. Yang, Y., Pedersen, J.O.: A comparative study on feature selection in text categorization. In: Fisher, D.H. (ed.) Proceedings of Fourteenth International Conference on Machine Learning, pp. 412-420 (1997)

15. Vapnik, V.N.: Statistical learning theory. Wiley, Chichester (1998)

16. Zadrozny, B.: Learning and evaluating classifiers under sample selection bias. In: Proceedings of the Twenty-First International Conference on Machine Learning (2004)

\section{Appendix: Data Set Composition}

There are too many sub-categories in Reuters-21578, so we ignore composition details of the last three data sets, which are from Reuters-21578. The detailed composition of the data sets from other two collections are completely shown below:

\begin{tabular}{|c|c|c|c|c|c|}
\hline Source & Data Set & Train/Test & Positive & Negative & \# of examples \\
\hline \multirow{4}{*}{$\begin{array}{l}\text { SRAA } \\
\text { (s.) }\end{array}$} & \multirow[t]{2}{*}{ s.ds 1} & train & simauto & simaviation & 8,000 \\
\hline & & test & realauto & realaviation & 8,000 \\
\hline & \multirow[t]{2}{*}{ s.ds2 } & train & realaviation & simaviation & 8,000 \\
\hline & & test & realauto & simauto & 8,000 \\
\hline \multirow{12}{*}{$\begin{array}{c}20 \\
\text { Newsgroups } \\
\text { (n.) }\end{array}$} & \multirow[t]{2}{*}{ n.ds3 } & train & $\begin{array}{c}\text { rec.autos } \\
\text { rec.motorcycles }\end{array}$ & $\begin{array}{l}\text { talk.politics.guns } \\
\text { talk.politics.misc }\end{array}$ & 3669 \\
\hline & & test & $\begin{array}{l}\text { rec.sport.baseball } \\
\text { rec.sport.hockey }\end{array}$ & $\begin{array}{l}\text { talk.politics.mideast } \\
\text { talk.religion.misc }\end{array}$ & 3561 \\
\hline & \multirow[t]{2}{*}{ n.ds 4} & train & $\begin{array}{c}\text { rec.autos } \\
\text { rec.sport.baseball }\end{array}$ & $\begin{array}{c}\text { sci.med } \\
\text { sci.space }\end{array}$ & 3,961 \\
\hline & & test & $\begin{array}{l}\text { rec.motorcycles } \\
\text { rec.sport.hockey } \\
\end{array}$ & $\begin{array}{c}\text { sci.crypt } \\
\text { sci.electronics } \\
\end{array}$ & 3,954 \\
\hline & \multirow[t]{2}{*}{ n.ds5 } & train & $\begin{array}{c}\text { comp.graphics } \\
\text { comp.sys.mac.hardware } \\
\text { comp.windows.x }\end{array}$ & $\begin{array}{l}\text { talk.politics.mideast } \\
\text { talk.religion.misc }\end{array}$ & 4,482 \\
\hline & & test & $\begin{array}{l}\text { comp.os.ms-windows.misc } \\
\text { comp.sys.ibm.pc.hardware }\end{array}$ & $\begin{array}{l}\text { talk.politics.guns } \\
\text { talk.politics.misc }\end{array}$ & 3,652 \\
\hline & \multirow[b]{2}{*}{ n.ds 6} & train & $\begin{array}{c}\text { comp.graphics } \\
\text { comp.os.ms-windows.misc } \\
\end{array}$ & $\begin{array}{c}\text { sci.crypt } \\
\text { sci.electronics }\end{array}$ & 3,930 \\
\hline & & test & $\begin{array}{c}\text { comp.sys.ibm.pc.hardware } \\
\text { comp.sys.mac.hardware } \\
\text { comp.windows.x }\end{array}$ & $\begin{array}{c}\text { sci.med } \\
\text { sci.space }\end{array}$ & 4,900 \\
\hline & \multirow[t]{2}{*}{ n.ds7 } & train & $\begin{array}{c}\text { comp.graphics } \\
\text { comp.sys.ibm.pc.hardware } \\
\text { comp.sys.mac.hardware }\end{array}$ & $\begin{array}{l}\text { rec.motorcycles } \\
\text { rec.sport.hockey }\end{array}$ & 4,904 \\
\hline & & test & $\begin{array}{c}\text { comp.os.ms-windows.misc } \\
\text { comp.windows.x }\end{array}$ & $\begin{array}{c}\text { rec.autos } \\
\text { rec.sport.baseball }\end{array}$ & 3,949 \\
\hline & \multirow[t]{2}{*}{ n.ds 8} & train & $\begin{array}{c}\text { sci.electronics } \\
\text { sci.med }\end{array}$ & $\begin{array}{l}\text { talk.politics.misc } \\
\text { talk.religion.misc }\end{array}$ & 3,374 \\
\hline & & test & $\begin{array}{l}\text { sci.crypt } \\
\text { sci.space }\end{array}$ & $\begin{array}{l}\text { talk.politics.guns } \\
\text { talk.politics.mideast }\end{array}$ & 3,828 \\
\hline \multirow{6}{*}{$\begin{array}{c}\text { Reuters } \\
- \\
21578 \\
\text { (r.) }\end{array}$} & \multirow[t]{2}{*}{ r.ds9 } & train & orgs. ${ }^{*}$ & places. ${ }^{*}$ & 1,078 \\
\hline & & test & orgs. ${ }^{*}$ & places. ${ }^{*}$ & 1,080 \\
\hline & \multirow[t]{2}{*}{ r.ds 10} & train & people.* & places.* & 1,239 \\
\hline & & test & people.* & places. ${ }^{*}$ & 1,210 \\
\hline & \multirow[t]{2}{*}{ r.ds11 } & train & orgs. ${ }^{*}$ & people.* & 1,016 \\
\hline & & test & orgs. ${ }^{*}$ & people.* & 1,046 \\
\hline
\end{tabular}

